\title{
An Incident of a Massive Pulmonary Embolism following Acute Aortic Dissection. A Case Report
}

\author{
Sofia Fernandes ${ }^{1 *}$, Mariana Rodrigues ${ }^{1}$, Catarina Barreiros ${ }^{1}$, Hugo Côrte-Real2 ${ }^{2}$, Ricardo \\ Ferreira², Ângelo Nobre² \\ 1 Department of Anesthesiology, Centro Hospitalar Universitario Lisboa Norte EPE, Lisboa, Portugal \\ 2 Department of Cardiothoracic Surgery, Centro Hospitalar Universitario Lisboa Norte EPE, Lisboa, Portugal
}

\begin{abstract}
Acute aortic dissection and acute pulmonary embolism are two life-threatening emergencies. The presented case is of an 81-year-old man who has been diagnosed with an acute Stanford type A aortic dissection and referred to a tertiary hospital for surgical treatment. After a successful aortic repair and an overall favourable postoperative recovery, he was diagnosed with cervical and upper extremity deep vein thrombosis and was anticoagulated accordingly. He later presented with massive bilateral pulmonary embolism.
\end{abstract}

Keywords: aortic dissection, pulmonary embolism, anticoagulation

Received: 14 September 2020 / Accepted: 29 December 2020

\section{INTRODUCTION}

Acute aortic dissection is a rare condition but the most common of the aortic catastrophes with a significant mortality rate if not appropriately diagnosed and treated $[1,2]$. Acute Stanford Type A aortic dissection requires emergent surgery. Acute pulmonary embolism is also a potentially life-threatening emergency, and the initiation of suitable therapeutic interventions relies on an appropriate identification [3].

Coexistence of pulmonary embolism and acute type A aortic dissection is extremely rare, with only a few reported incidents $[4,5]$. The pathophysiology behind concomitant pulmonary embolism and acute aortic dissection involves an external mechanical compression of the pulmonary artery from ascending aortic dissection in association with coagulation derangements also caused by the aortic dissection.

The presented case is of an elderly man who underwent surgery for acute Stanford Type A aortic dissection and during the postoperative period was found simultaneously to have a pulmonary embolism. Although fibrinolytic therapy was contraindicated, con- sidering risks and benefits, it may have been lifesaving in this case.

\section{CASE PRESENTATION}

An 81-year-old male patient, with a history of systemic hypertension, hyperuricemia, nephrectomy for urothelial carcinoma (2009) and chronic renal disease was admitted to a secondary care hospital (Unidade Local de Saúde do Litoral Alentejano, EPE, Santiago do Cacém, Portugal) on Apr 21 $1^{\text {st }}, 2020$, with chest pain radiating to the neck and inter-scapular region. On admission, physical examination revealed no signs of difficulty breathing, oxygen saturation of $98 \%$ at room air and no arterial blood gas abnormalities. He presented with a normotensive profile, mean blood pressure 78-88 $\mathrm{mmHg}$, and a heart rate of 70 beats/min. An electrocardiogram was taken and showed no evidence of myocardial ischemia. A computed tomographic thoracic angiography was performed (Phillips ${ }^{\circledR}$ Brilliance 64 ; intravenous Iomeron ${ }^{\circledR}$ contrast, $80 \mathrm{~mL}$ ) revealing an acute Stanford type A aortic dissection (Figure 1-A) with a 78 $\mathrm{mm}$ saccular aneurysm of the aortic arch and a $17 \mathrm{~mm}$ accumulation of pericardial effusion (Figure 1-B). 

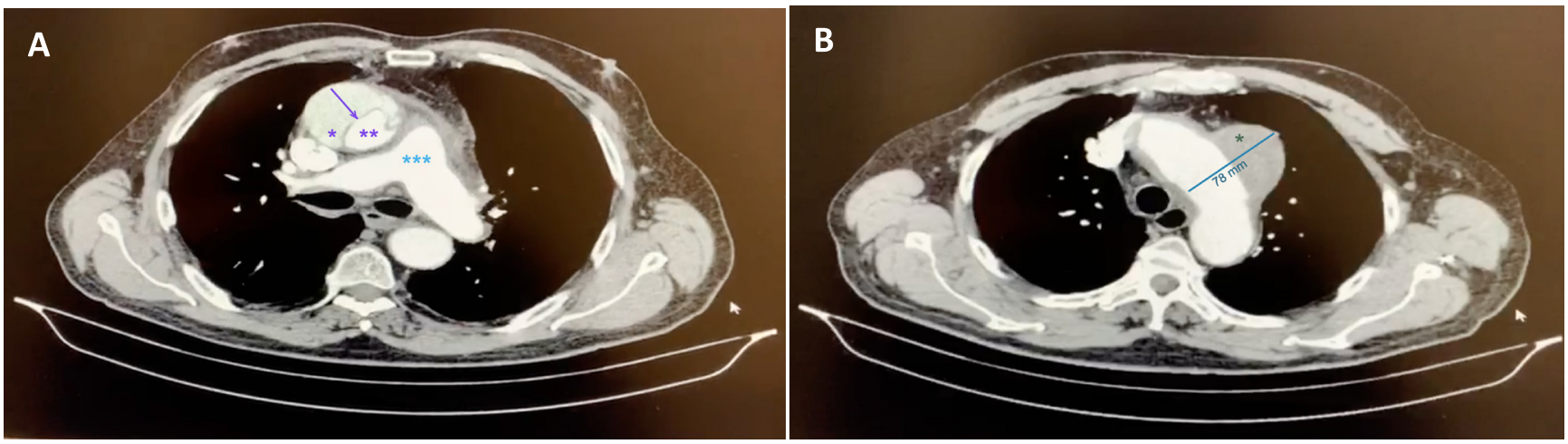

Fig. 1. A. Preoperative thoracic computed tomographic angiography: acute Stanford type A aortic dissection with ascending intimal flap (arrow). The main pulmonary artery $(* * *)$ and the left and right branches were normally enhanced with contrast medium. (*true lumen aorta; **false lumen). B. Preoperative thoracic computed tomographic angiography: aortic arch saccular aneurysm (*).

The main pulmonary artery and the left and right branches had normal enhancement with contrast medium. The patient had no history of obstructive sleep apnoea or respiratory failure. He was subsequently transferred to a tertiary hospital (Hospital de Santa Maria, Centro Hospitalar Universitário Lisboa Norte, Lisbon, Portugal) on Apr 23 $3^{\text {rd }}$ to receive adequate surgical treatment.

On admission, the patient was hemodynamically stable; his blood pressure was 99-123/58-76 mmHg, and his heart rate was $72-85$ beats/min. He was being given labetalol (SALF S.p.A., Bergamo, Italy) intravenous continuous infusion of $0.3 \mathrm{mg} / \mathrm{min}$.

No information regarding respiratory function was obtained. His arterial blood gas on a $40 \%$ Venturi mask was $\mathrm{pH} 7.40, \mathrm{PaCO}_{2} 42.4 \mathrm{mmHg}, \mathrm{PaO}_{2} 69.9 \mathrm{mmHg}$, $\mathrm{HCO}_{3} 25.4 \mathrm{mmol} / \mathrm{L}, \mathrm{BE}-0.2 \mathrm{mmol} / \mathrm{L} ; \mathrm{SaO}_{2} 94.1 \%$, demonstrating type 1 respiratory failure. A complete blood count showed the following: Hgb $12.8 \mathrm{~g} / \mathrm{dL}$; Htc $36.9 \%$; platelet count $115.000 / \mu \mathrm{L}$; activated partial thromboplastin time (aPTT) 26.2/29.0 seconds; International Normalized Ratio (INR) 1.21; fibrinogen 474 $\mathrm{mg} / \mathrm{dL}$; serum creatinine $1.89 \mathrm{mg} / \mathrm{dL}$; and troponin $\mathrm{T}$ (cTnT-hs) $94 \mathrm{ng} / \mathrm{L}$.

A four-lumen central line in the right internal jugular vein and a left radial arterial line were placed under ultrasound guidance. Preoperative urinary output was approximately $1 \mathrm{~mL} / \mathrm{kg} / \mathrm{h}$.

Intraoperative trans-oesophageal echocardiography (Phillips ${ }^{\circledast}$ Epiq 7G; X7-2T probe) revealed an enlarged ascending aorta of $60 \mathrm{~mm}$, an intimal flap dissection distal to the sino-tubular junction, an aortic arch dilatation of approximately $50 \mathrm{~mm}$, with a $70 \mathrm{~mm}$ saccular aneurysm and spontaneous thrombosis in the distal arch at the emergence of the left subclavian artery, a small $12 \mathrm{~mm}$ accumulation of pericardial effusion and normal biventricular function.

An ascending aorta and aortic arch replacement with a woven polyester vascular graft $(30 \mathrm{~mm}$, FlowWeave Bioseal JOTEC ${ }^{\circledast}$ ) and en-bloc replacement of the supraaortic vessels as an island patch and endarterectomy of the innominate artery were carried out.

Surgery was accomplished under deep hypothermic cardiopulmonary arrest at $25^{\circ} \mathrm{C}$ with selective anterograde cerebral perfusion. Extracorporeal circulation with right axillary arterial and right atrium appendage venous cannulation was maintained for ninety-four minutes, aortic cross-clamping for seventy-two minutes and deep hypothermic cardiopulmonary arrest for forty-two minutes.

A high $\mathrm{FiO}_{2}$ of $0.8-1.00$ was required during the procedure. The patient was weaned off bypass uneventfully.

He was subsequently transferred to the intensive care unit mechanically ventilated, on pressure regulated volume control mode (PRVC) with a tidal volume of 7 $\mathrm{mL} / \mathrm{kg}$, respiratory rate $18 / \mathrm{min}$, PEEP $8 \mathrm{~cm} \mathrm{H}_{2} \mathrm{O}, \mathrm{FiO}_{2}$ 1.0, with intravenous continuous-infusion noradrenaline (Generis SA Farmacêutica, Amadora, Portugal) at a dose of $0.35 \mu \mathrm{g} / \mathrm{kg} / \mathrm{min}$ and continuous intravenous infusion of dobutamine (Generis SA Farmacêutica, Amadora, Portugal) at a dose of $4.7 \mu \mathrm{g} / \mathrm{kg} / \mathrm{min}$.

At the time of admission to the intensive care unit, the patient presented with atrial fibrillation with a rapid ventricular response and coagulopathy-related diffuse bleeding. Laboratory test results showed the following: an INR 1.38, aPTT 37.0/29.0 seconds, fibrinogen 245 $\mathrm{mg} / \mathrm{dL}, \mathrm{Hgb} 8.5 \mathrm{~g} / \mathrm{dL}$, platelet count $91.000 / \mu \mathrm{L}$ and Ddimer $1041 \mu \mathrm{g} / \mathrm{L}$. Thromboelastography gave the following results: EXTEM-CT 73 seconds (38-79), A10 50 
mm (43-65) and MCF 59 mm (50-72); FIBTEM-CT 62 seconds; A10 $16 \mathrm{~mm}$ and MCF $18 \mathrm{~mm}$; platelet-TEM AUC 49 (55-154), A6 13 (14-36) and MS 4 (5-14).

Accordingly, $4 \mathrm{~g}$ of fibrinogen, one platelet concentrate, $800 \mathrm{~mL}$ of fresh frozen plasma and two units of packed red blood cells were administered.

Arterial blood gas test measures were recorded as follows: $\mathrm{pH}$ 7.39, $\mathrm{PaCO}_{2} 41.7 \mathrm{mmHg}, \mathrm{PaO}_{2} 74.0$ $\mathrm{mmHg}, \mathrm{HCO}_{3} 24.5 \mathrm{mmol} / \mathrm{L}$, BE $1.2 \mathrm{mmol} / \mathrm{L} ; \mathrm{SaO}_{2}$ $95.0 \%$, lactate $25 \mathrm{mg} / \mathrm{dL}$. Ventilation was maintained with pressure-regulated volume control (PRVC) mode at a tidal volume of $6 \mathrm{~mL} / \mathrm{kg}$, respiratory rate of $20 / \mathrm{min}$, $\mathrm{FiO}_{2}$ 1.0, PEEP $12 \mathrm{cmH}_{2} \mathrm{O}$, obtaining a static compliance of $32 \mathrm{~mL} / \mathrm{cmH}_{2} \mathrm{O}$ and $\mathrm{PaO}_{2} / \mathrm{FiO}_{2}$ ratio of 74 .

A diagnosis of acute respiratory distress syndrome (ARDS) from multifactorial causes was made.

The patient was kept sedated with an intravenous continuous-infusion of propofol (DIPRIVAN ${ }^{\odot}$, Aspen Pharmacare, Durban, South Africa) at a dose of 2.5 $\mathrm{mg} / \mathrm{kg} / \mathrm{h}$ and an intravenous continuous-infusion of remifentanil (ULTIVA ${ }^{\odot}$, Aspen Pharmacare, Durban, South Africa) at a dose of $5 \mu \mathrm{g} / \mathrm{kg} / \mathrm{h}$. He was pharmacologically paralyzed with an intravenous bolus of rocuronium (ESMERON ${ }^{\odot}$, Merck Sharp \& Dohme, Lda., Paço de Arcos, Portugal) at a dose of $0.6 \mathrm{mg} / \mathrm{kg}$.

The patient required progressively higher vasopressor and inotropic support of noradrenaline $0.40 \mathrm{mcg} /$ $\mathrm{kg} / \mathrm{min}$; dobutamine $9 \mu \mathrm{g} / \mathrm{kg} / \mathrm{min}$ and a continuous infusion of adrenaline (Labesfal Farma, Tondela, Portugal) at a dose of $0.2 \mu \mathrm{g} / \mathrm{kg} / \mathrm{min}$.

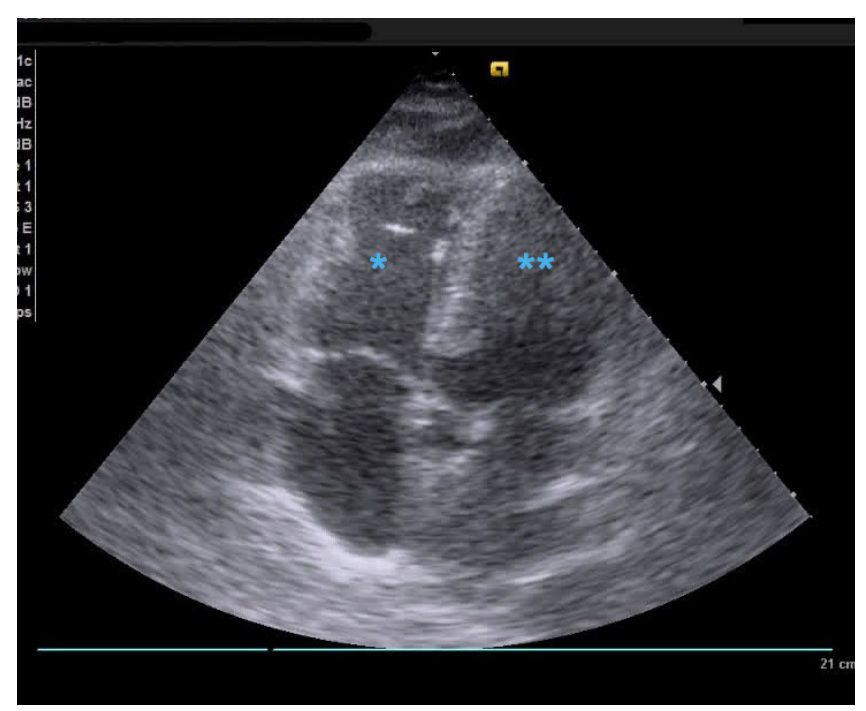

Fig. 2. Transthoracic echocardiography apical fivechamber view: right ventricular enlargement $(*)$ and left ventricle $(* *)$.
On the second day postoperatively, transthoracic echocardiography demonstrated a dysfunctional and severely enlarged right ventricle with tricuspid annular plane systolic excursion of $12 \mathrm{~mm}$ and a flattened septum with preserved left ventricular function (Figure 2).

A right ventricular infarction, troponin $\mathrm{T}$ level 704 ng/L, was hypothesized, with ARDS and cardiac surgery as possible mechanisms. Right ventricular function improved with inotropic support and the management of ARDS during the five following days.

As the patient's oxygenation improved with supportive care, oxygen requirements were reduced from 0.8 to 0.5 of $\mathrm{FiO}_{2}$ (pH 7.40; $\mathrm{PaCO}_{2} 38.9 \mathrm{mmHg} ; \mathrm{PaO}_{2} 112$ $\mathrm{mmHg} ; \mathrm{SaO}_{2} 99 \%$; $\mathrm{HCO}_{3} 24.7 \mathrm{mmol} / \mathrm{L} ; \mathrm{BE} 0.4$ ).

Sedation was gradually reduced for a neurological examination. Neurologic examination revealed left hemiparesis, with significant brachial involvement. Cranial computed tomography demonstrated corticosubcortical hypodensities in the right middle and ascending frontal circumvolutions and in the right paramedian posterior parietal topography translating areas of subacute ischemia from thromboembolic sources.

On the $4^{\text {th }}$ postoperative day, the patient developed asymmetrical left upper extremity swelling with a circumferential difference of about $2 \mathrm{~cm}$ to the contralateral arm, involving the upper arm, forearm and hand. Both limbs were warm to touch, with a bilateral radial pulse and normal capillary refill times.

Ultrasound revealed an occlusive thrombus of the left internal jugular, subclavian, axillary and brachial veins. Laboratory tests results showed an INR 1.30, aPTT 30.5/29.0 seconds, fibrinogen $678 \mathrm{mg} / \mathrm{dL}$ and Ddimer $1179 \mu \mathrm{g} / \mathrm{L}$. There was no evidence of lower limb deep vein thrombosis.

After multidisciplinary discussions between attending physicians, and risk assessments being carried out, a decision was taken, despite the known cerebral ischemic events, to prescribe anticoagulation therapy. Subcutaneously daily enoxaparin (LOVENOX ${ }^{\odot}, \mathrm{Sa}-$ nofi S.A., Paris, France) of $1.5 \mathrm{mg} / \mathrm{kg}$ was initiated. Improvement of the swelling and left hemiparesis followed three days after.

The patient improved substantially with diminishing vasopressor and inotrope requirements, and on the eleventh postoperative day, successful extubation was possible. His Glasgow Coma Score was 14 (E4V4M6), and he was referred to rehabilitation. 
On the $12^{\text {th }}$ postoperative day, following a course of physical and respiratory rehabilitation, the patient presented with motor agitation, acute peripheral oxygen desaturation, bradycardia (heart rate 35 beats/min), hypotension (BP 74/31 mmHg) and diaphoresis.

Despite treatment, he evolved to pulseless electrical activity. Advanced cardiac life support was initiated. The patient was re-intubated, and a return of spontaneous circulation was achieved after seven minutes. A point-of-care transthoracic echocardiogram demonstrated a dilated right ventricle with severe dysfunction and interventricular septum rectification. A primary diagnosis of pulmonary embolism was suspected.

Contrast-enhanced thoracic tomography revealed extensive, bilateral thromboembolism of the main pulmonary artery and its lobar branches (Figure 3-A). A periprosthetic hematoma with a pseudoaneurysm with a maximum thickness of $3.8 \mathrm{~cm}$ was observed arising from the innominate artery. This was causing a slight compression of the brachiocephalic venous trunk filiform pathway (Figure 3-B).

The presence of a massive pulmonary embolism was an indication for treatment with fibrinolysis. However, this approach was not considered appropriate due to the recent acute aortic dissection and cerebral stroke. Supportive inotropic treatment, ventilation and anticoagulation with enoxaparin were continued.

The patient remained in refractory obstructive shock with severe perfusion impairment and progressive biventricular failure.

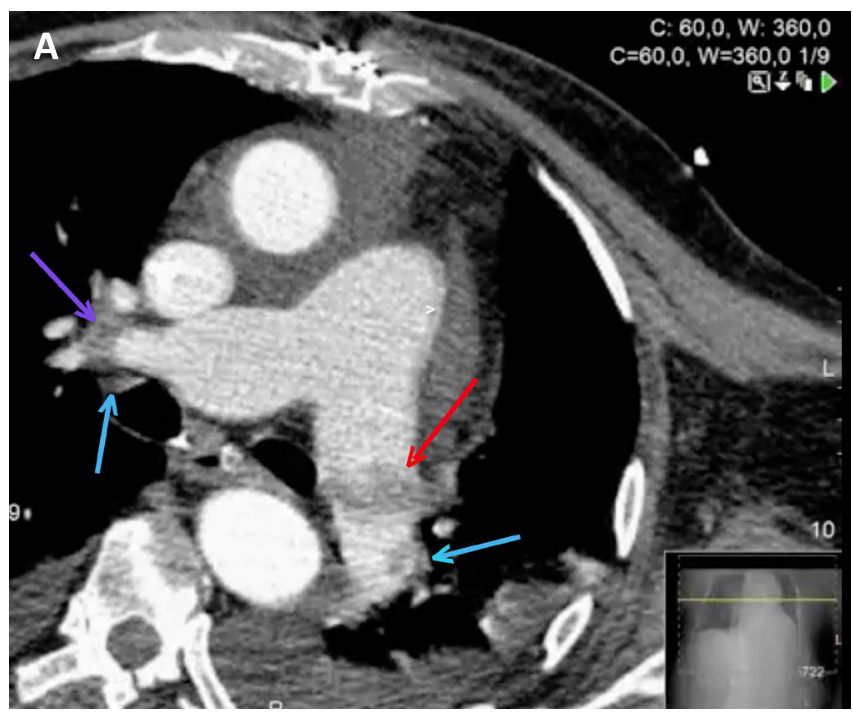

Fig. 3. A. Contrast-enhanced thoracic CT showing extensive, bilateral thromboembolism of the right (purple arrow) and left pulmonary artery (red arrow) branches and its lobar branches (blue arrows). B. Periprosthetic hematoma from the innominate artery with a pseudoaneurysm $(*)$ conditioning a slight impression of the brachiocephalic venous trunk filiform path (red arrow).

Given the poor prognosis, maintaining organ support was considered futile and active therapeutic measures were suspended. Cardiac arrest was confirmed shortly afterwards.

\section{Discussion}

Acute aortic dissection is one of the most common aortic catastrophes with extremely high morbidity and mortality rates. The mortality rate of acute Stanford type A aortic dissection approaches $26 \%$ with surgical therapy and has dropped to $12 \%$ during the last decade due to surgical advances and improved postoperative management [6,7]. However, in-hospital surgical mortality for acute aortic dissection is approximately $25 \%$ [2].

Pulmonary embolism is a relatively common cardiovascular emergency, which may result in acute lifethreatening but potentially reversible right ventricular failure due to obstruction of the pulmonary arterial bed [8]. During the first hours of admission, the current patient's transthoracic echocardiogram demonstrated right ventricular enlargement with dysfunction. We hypothesized that right ventricular infarct, i.e. a right coronary artery hematoma versus dissection, ARDS and cardiac surgery were the most likely mechanisms.

Right ventricular dysfunction with increasing inotropic and vasopressor support and an induced prothrombotic state due to transfusion of allogeneic blood products, as substantiated with high serum levels of

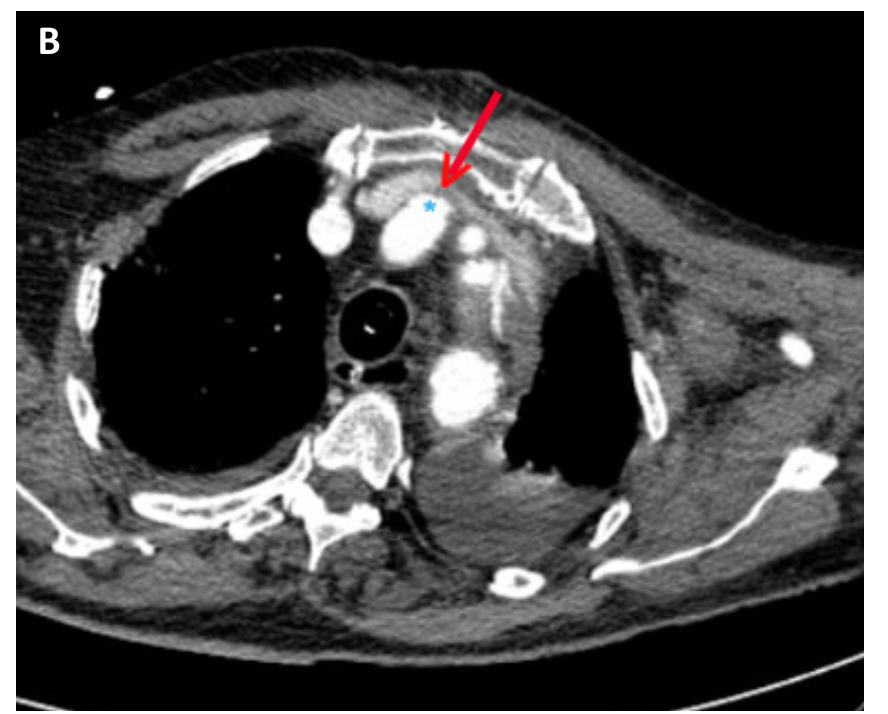


fibrinogen $678 \mathrm{mg} / \mathrm{dL}$ and D-dimer $1179 \mu \mathrm{g} / \mathrm{L}$, could suggest a pulmonary embolism.

Pulmonary embolism after aortic dissection is uncommon. The prognosis of medical recovery may be far worse, and complicates patient management considerably $[9,10]$. Aortic dissection pathophysiological consequences involve an immune response, activation of the coagulation system and possible mass-compression effects, all of which promote thrombus formation.

Guan et al. (2016) and Liu et al. (2017) have reported a systemic activation of haemostatic system and anticoagulant pathway inhibition in a group of patients who underwent aortic arch surgery for acute aortic dissection. The phenomenon may contribute towards microvascular and macrovascular thrombosis, even before surgery.

Furthermore, aortic surgery is associated with an increased risk of venous thromboembolism owing to hypercoagulability due to increased thrombin generation and activity and fibrin turnover in aortic arch surgery and extracorporeal circulation [11-13].

Surgery and prolonged immobilization are also wellknown thromboembolism risk factors. Despite routine thromboembolism prophylaxis, a pulmonary embolism frequently occurs.

The current patient presented with acute aortic dissection followed by a positive evolution during the first eleven postoperative days despite being affected by ARDS, venous thrombosis and cerebral ischemic stroke.

Postoperative stroke incidence after acute aortic dissection surgery has been reported to be 2 to $16 \%$ $[14,15]$. Dumfarth et al. (2018) identified preoperative cardiopulmonary resuscitation and malperfusion syndromes as independent predictors for postoperative stroke [15]. Our patient was not in a critical state at presentation, and surgical arterial cannulation was performed through the right axillary artery.

The patient's thromboembolic risk was high. Therapeutic anticoagulation was initiated after an acute left extremity deep venous thrombosis followed by a noticeable clinical improvement.

Despite treatment, the patient developed a massive pulmonary embolism leading to cardiac arrest. It was assumed that the pulmonary embolism originated from thrombus dislodgement from the left arm and the cervical deep-venous system, migrating to the pulmonary circulation, and leading to obstructive shock.
The hematoma from the innominate artery compressing the brachiocephalic trunk, contributed to the upper left extremity deep venous thrombosis, apart from aortic inflammation, coagulation system activation, transfusion of allogeneic products and thrombosis generation from acute aortic dissection and extracorporeal circulation.

Urgent surgery for acute aortic dissection in patients over 80 and older remains a controversial issue because of its high surgical risk. Reports describe excellent surgical results in elderly patients $[16,17]$. However, other reports show that patients aged 80 and older were at increased risk of hospital mortality $[18,19]$. In our centre, there is no age restriction to surgical repair on acute Stanford type A AD in the elderly, except in the case of preoperative cardiac arrest.

In the present case report, factors such as age, chronic renal failure, ARDS, stroke and pulmonary embolism may have contributed to the negative outcome.

\section{a CONCLUSION}

Although fibrinolytic therapy can be a lifesaving option, in this case, it was contraindicated considering the dissection, rupture and high bleeding risk in a patient with ischemic stroke. Management of such patients remains a challenge in everyday clinical practice.

\section{DEFERENCES}

1. Berretta P, Patel HJ, Gleason TG, et al. IRAD experience on surgical type $A$ acute dissection patients: results and predictors of mortality. Ann Cardiothorac Surg 2016;5(4):346-351.

2. Poon SS, Field M. Target mortality for repair of acute type A dissection. J Thorac Cardiovasc Surg 2019 Apr;157(4):e113-e115.

3. Paul JD, Cifu AS. Management of Acute Pulmonary Embolism. JAMA. 2020;324(6):597-598.

4. Nakamura K, Orii K, Hanai M, Abe T, Haida H. Management of acute pulmonary embolism after acute aortic dissection surgery. J Cardiol Cases. 2020 Aug 3;22(4):195-197.

5. Leu, HB, Yu WC. Images in cardiology: massive pulmonary embolism in a patient with type $A$ aortic dissection. Clin Cardiol. 2005; 28:53.

6. Conway BD, Stamou SC, Kouchoukos NT, et al. Improved clinical outcomes and survival following repair of acute type $A$ aortic dissection in the current era. Interact Cardiovasc Thorac Surg. 2014;19(6):971-6.

7. Omura A, Miyahara S, Yamanaka K, et al. Early and late outcomes of repaired acute DeBakey type I aortic dissection 
after graft replacement. J Thorac Cardiovasc Surg. 2016; 151:341-8

8. Konstantinides SV, Meyer G, Becattini C, et al. 2019 ESC Guidelines for the diagnosis and management of acute pulmonary embolism developed in collaboration with the European Respiratory Society (ERS): The Task Force for the diagnosis and management of acute pulmonary embolism of the European Society of Cardiology (ESC). Eur Heart J 2020 Jan 21;41(4):543-603.

9. Blanke $P$, Apfaltrer $P$, Ebersberger $U$, et al. CT detection of pulmonary embolism and aortic dissection. Cardiol Clin 2012; 30:10316.

10. SemizOysu A, Okur A, Sahin S. Pulmonary multislice computed tomography findings in acute aortic dissection. J Thorac Dis 2012; 4:4859.

11. Guan X, Li J, Gong M, et al. The hemostatic disturbance in patients with acute aortic dissection: a prospective observational study. Medicine 2016; 95: e4710.

12. Guan $X$, Wang $X$, Liu YY, et al. Changes in the hemostatic system of patients with acute aortic dissection undergoing aortic arch surgery. Ann Thorac Surg. 2016; 101:945-51.

13. Liu Y, Han L, Li J, et al. Consumption coagulopathy in acute aortic dissection: principles of management. J Cardiothorac
Surg. 2017; 12:50.

14. Nishida H, Tabata M, Fukui T, et al. A systematic approach to improve the outcomes of type A aortic dissection. J Thorac Cardiovasc Surg 2017; 154:89-96.

15. Dumfarth J, Kofler M, Stastny L, et al. stroke after emergent surgery for acute type A aortic dissection: predictors, outcome and neurological recovery. Eur J Cardiothorac Surg 2018; 53:1013-20.

16. Tang GH, Malekan R, Yu CJ, et al. surgery for acute type A aortic dissection in octogenarians is justified. J Thorac Cardiovasc Surg 2013;145: S186-90.

17. Matsushita A, Tabata M, Fukui $T$, et al. Outcomes of contemporary emergency open surgery for type $A$ acute aortic dissection in elderly patients. J Thorac Cardiovasc Surg 2014; 147:290-4.

18. Ohnuma T, Shinjo D, Fushimi K. Hospital mortality of patients aged 80 and older after surgical repair for type $A$ acute aortic dissection in Japan. Medicine (Baltimore) 2016 Aug; 95(31): e4408.

19. Trimarchi S, Eagle KA, Nienaber CA, et al. Role of age in acute type $A$ aortic dissection outcome: Report from the International Registry of Acute Aortic Dissection (IRAD). J Thorac Cardiovas Surg 2010; 140:784-9. 